\title{
Third time re-irradiation of liver metastasis with robotic radiosurgery: a case series
}

\author{
Debnarayan Dutta1, Sathiya Krishnamoorthy², Rupa Das ${ }^{1}$, Ram Madhavan$^{1}$, Haridas Nair ${ }^{1}$, Raghavendra \\ Holla $^{1}$ \\ 1Amrita Institute of Medical Sciences and Research Centre, Kochi, Kerala 682041, India. \\ ${ }^{2}$ Department of Radiation Oncology, Global Hospital, Chennai 600035, India.
}

Correspondence to: Debnarayan Dutta, Amrita Institute of Medical Sciences and Research Centre, Kochi, Kerala 682041, India. E-mail:duttadeb07@gmail.com

How to cite this article: Dutta D, Krishnamoorthy S, Das R, Madhavan R, Nair H, Holla R. Third time re-irradiation of liver

Received: 5 Feb 2019 First Decision: 17 Apr 2019 Revised: 20 Jun 2019 Accepted: 20 Jun 2019 Published: 11 Jul 2019

Science Editor: Guang-Wen Cao Copy Editor: Cai-Hong Wang Production Editor: Jing Yu

\begin{abstract}
Re-irradiation (Re-RT) in liver tumours is rarely reported owing to poor tolerance of liver and high incidence of radiation induced liver disease incidence. Fiducial based robotic radiosurgery allows to deliver high dose radiation to the liver tumour and restricts the dose to healthy uninvolved liver, thereby increasing the potential for Re-RT. Tolerance to radiation is low for entire liver and hence re-radiation is a challenge. On the other hand, as regenerative potential of hepatocytes is rapid, replacement of necrotic liver tissue occurs with regenerated hepatocytes. These regenerated hepatocytes are radiation naïve, do not have "memory" of radation therapy treatment and hence have potential of Re-RT. We are reporting a series of two breast cancer patients presented with liver oligometastasis treated with fiducial based CyberKnife system (CK). Both the patients were treated multiple times with CK and had long-term survival (> 2 years) without any clinical features of radiation induced liver injury. Appropriately selected patients are suitable for multiple sessions of CK for liver lesions with long-term outcome.
\end{abstract}

Keywords: Liver lesions, re-radiation, robotic radiosurgery

\section{INTRODUCTION}

Liver tissue has poor tolerance to radiation therapy (RT), hence RT was rarely considered for liver tumours. Mean liver dose as low as 15 Gy to the whole liver can cause injury to the liver [radiation induced liver disease (RILD)], presenting with anicteric hepatitis, ascites and progressive deterioration of liver

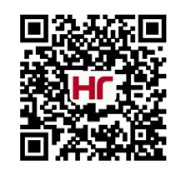


Table 1. Case report of breast cancer with liver metastasis at diagnosis and re-treated with radiosurgery

\begin{tabular}{|c|c|c|c|}
\hline Date & Event & Treatment & Investigations \\
\hline Sept 2014 & $\begin{array}{l}\text { Left Breast carcinoma } \\
\text { pT2N2M1 } \\
\text { ER/PR +ve; Her2neu +ve }\end{array}$ & Lumpectomy à Left MRM & $\begin{array}{l}\text { PETCT - Hypermetabolic liver metastases in Seg VII } \\
(\text { size }-2 \mathrm{~cm} \times 1.6 \mathrm{~cm})\end{array}$ \\
\hline Oct 2014 & SBRT (1st CK) & \multicolumn{2}{|c|}{ SBRT to Segment VII liver lesions - 45 Gy in 3 fractions, ( prescription to $88 \%$ isodose ) } \\
\hline Nov 2014 & Adjuvant chemotherapy & \multicolumn{2}{|c|}{ TCH -Taxol, carbolatin, Herceptin $\times 6$ cycles; Herceptin continued $\times 1$ yr } \\
\hline Mar 2015 & $\begin{array}{l}\text { Adjuvant radiation therapy } \\
\text { Hormonal therapy }\end{array}$ & \multicolumn{2}{|c|}{$\begin{array}{l}\text { EBRT to left chest wall and region nodes }-50 \mathrm{~Gy} / 25 \mathrm{fr} / 5 \mathrm{wk} \\
\text { Inj Goserelin and Tab Tamoxifen }\end{array}$} \\
\hline April 2016 & Restaging & \multicolumn{2}{|c|}{ PETCT - No significant metabolically active disease } \\
\hline Sept 2016 & Restaging & \multicolumn{2}{|c|}{ PETCT - Interval new hepatic metastases in segment VIII $(3 \mathrm{~cm} \times 2.4 \mathrm{~cm})$} \\
\hline Sept 2016 & SBRT (2nd CK) & \multicolumn{2}{|c|}{ SBRT to Seg VIII liver lesion - 45 Gy/3 fr ( prescription at $88 \%$ iso-dose) } \\
\hline Oct 2016 & Change hormonal therapy & \multicolumn{2}{|c|}{ Letrazole, Herceptin restarted, Inj Goserlin continuing } \\
\hline March 2018 & Restaging & \multicolumn{2}{|c|}{$\begin{array}{l}\text { PETCT - Abnormal increased uptake in subtle hypodensity in segment VI of liver } \\
\text { (SUVMax 6.5) }\end{array}$} \\
\hline April 2018 & SBRT (3rd CK) & \multicolumn{2}{|c|}{$\begin{array}{l}\text { SBRT to seg VI liver lesion - } 50 \mathrm{~Gy} / 5 \mathrm{fr} \text {, prescription at } 88 \% \\
\text { Continuing Herceptin, Inj Goserlin }\end{array}$} \\
\hline Sep 2018 & Restaging & \multicolumn{2}{|c|}{ PETCT - liver lesion completely resolved. No other abnormality detected } \\
\hline
\end{tabular}

SBRT: stereotactic body radiotherapy; PETCT: positron emission tomography-computed tomography; EBRT: external beam radiation therapy

function $^{[1,2]}$. Only after high precision RT was introduced and clinicians had knowledge regarding partial liver radiation, RT gained momentum in the treatment of liver tumours. Stereotactic radiation therapy with real time liver tracking have helped in delivering safely high dose precise short course RT (radiosurgery) in liver tumours. However, the risk of liver injury is always a concern in treatment of liver tumours. In such a situation it is likely that re-irradiation (Re-RT) in liver tumours will be rarely reported.

Fiducial based robotic radiosurgery (CyberKnife, CK, Accuray ${ }^{\circ}$; Sunnyvale, CA) gives liberty to deliver high dose radiation to liver tumours and restrict dose to surrounding healthy liver cells, thereby increasing the potential for Re-RT ${ }^{[3,4]}$. Re-RT in liver tumours is a challenge, and needs active evaluation of possible toxicities before initiating the treatment. There are higher risks of liver decompensation and incidence of RILD. Toxicity and response to treatment after stereotactic body radiotherapy (SBRT) depends upon mean liver dose, amount of spared normal liver volume, previous treatment and modality of treatment ${ }^{[5]}$. On the other hand, regenerative potential of hepatocytes is rapid and rapidly proliferating hepatocytes replacement of necrotic liver tissue, those expected to have no "memory" of RT as they are naïve to radiation ${ }^{[2]}$. Hence, there is a potential for Re-RT in liver with rapid regeneration of hepatocytes ${ }^{[2]}$. We are reporting a series of two breast cancer patients presented with liver oligometastasis and are treated with fiducial based CyberKnife system (CK).

\section{CASE REPORT}

A forty-five year-old female was diagnosed in Sept 2014 with left breast cancer $(2 \mathrm{~cm} \times 2 \mathrm{~cm}$, Upper Outer Quadrant, mobile axillary nodes) [Table 1]. Metastatic workup with PET-CT revealed solitary metastasis in segment VII of liver $(2 \mathrm{~cm} \times 1.6 \mathrm{~cm}$, SUVmax -7). She had normal liver function (Child Pugh A) and viral markers were negative. She underwent Left Modified radical mastectomy and was subsequently treated with CK (45 Gy in 3 fractions, prescribed to 88\%, mean liver dose 681 cGy) in Oct 2014 [Figure 1]. Histopathology - Infiltrating ductal carcinoma (IDC), Gr III, 5/11 nodes positive, ER/PR +ve, Her2neu +ve. She received chemotherapy (Taxane based chemotherapy) followed by adjuvant RT to chest wall (45 Gy/25 fr/5 wk) and then received Trastuzumab for 1 year along with Tamoxifen (HT). Follow up PET scan in 2015 was complete response (CR) and no focal lesion seen in liver. Repeated PET scan (Sept 2016) showed a new solitary liver lesion $(3 \mathrm{~cm} \times 2.5 \mathrm{~cm}$, SUVmax -8$)$ in segment VIII. She was re-irradiated with CK (45 Gy/3 fr, $88 \%$ isodose, mean liver dose 771 cGy). Follow-up PET scan (Mar 2017) showed no evidence of disease and repeat scan in Nov 2017 showed complete regeneration of both segment VII and VIII region without any 


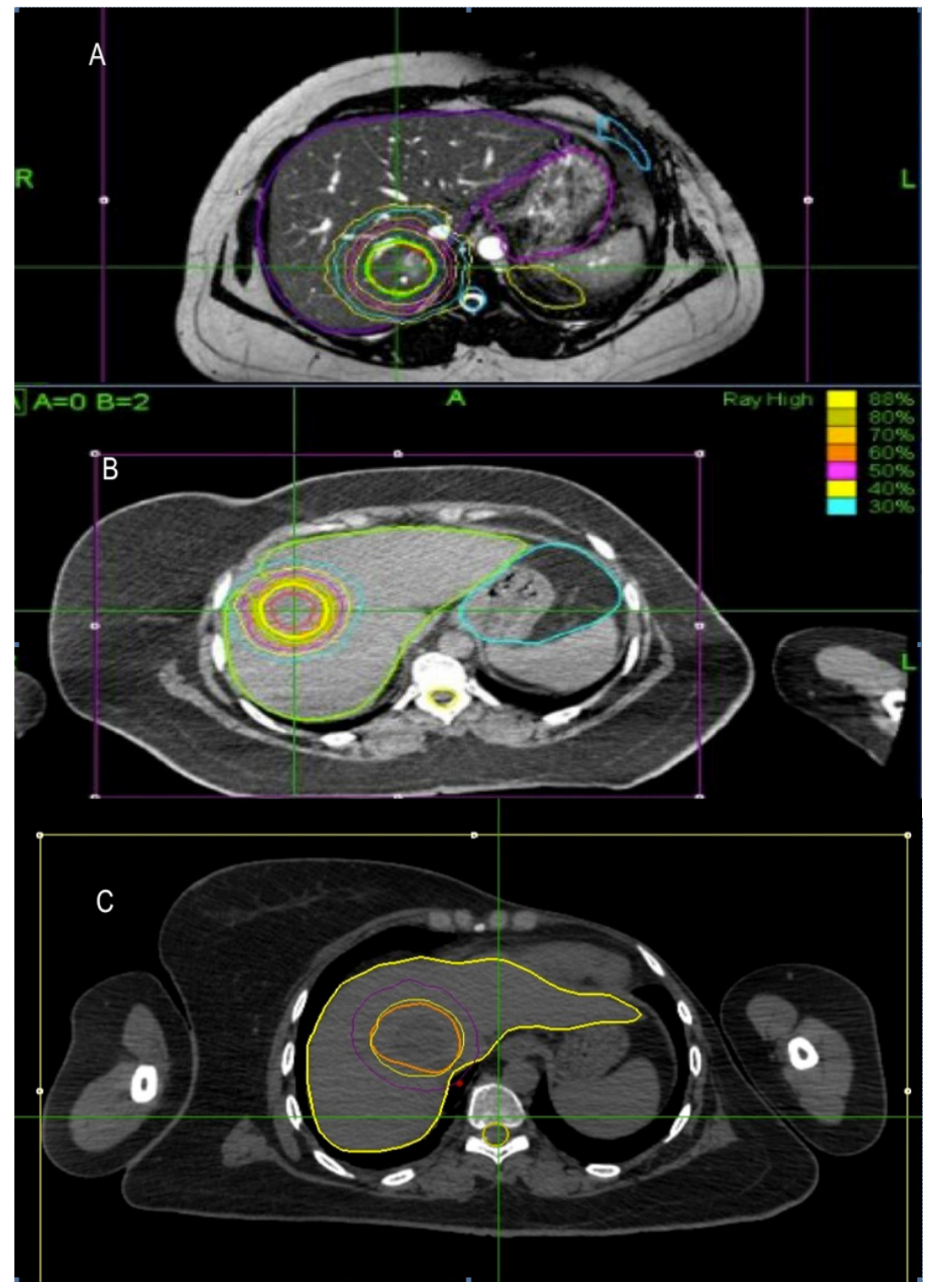

Figure 1. CyberKnife treatment plans. A: First CyberKnife plan ( $45 \mathrm{~Gy} / 3$ fr); B: Second CyberKnife plan ( $45 \mathrm{~Gy} / 3$ fr); C: Third CyberKnife plan (50 Gy/5 fr)

evidence of disease. Follow-up scan in March 2018 showed a new lesion in segment II $(2.5 \mathrm{~cm} \times 2.3 \mathrm{~cm})$. She was treated the third time with fiducial based SBRT 50 Gy/5 fr [(prescription 88\% isodose) three Accuray defined fiducials, distributed by Alphamed ${ }^{\circledR}$. Response evaluation scan (Sep 2018) showed significant regression of SUV uptake and mass in liver. She had no sign of radiation induced liver injury [Figure 2]. Three fiducials were placed close to the tumour under USG guidance by radiologist. Same fiducials were used for tracking during Re-RT. In situations where new lesion is in another lobe of liver, larger PTV margin ( $5 \mathrm{~mm}$ ) was given to the GTV.

A thirty-six year-old female was diagnosed with carcinoma right breast (cT1N1M0, IDC Gr III, ER/ PR +ve, Her2neu -ve) in Nov 2010, was treated with breast conservative surgery (BCS). She received adjuvant systemic therapy (FEC $\times 4$ and Docetaxel $\times 4$ cycle), followed by adjuvant loco-regional RT ( 45 Gy/25 fr/5 wk) [Table 2]. She was on periodic follow-up and had controlled disease until Feb 2014 when routine PET scan showed multiple liver lesions in both lobes, largest measuring $7 \mathrm{~cm} \times 8 \mathrm{~cm}$ in Seg VI/ 


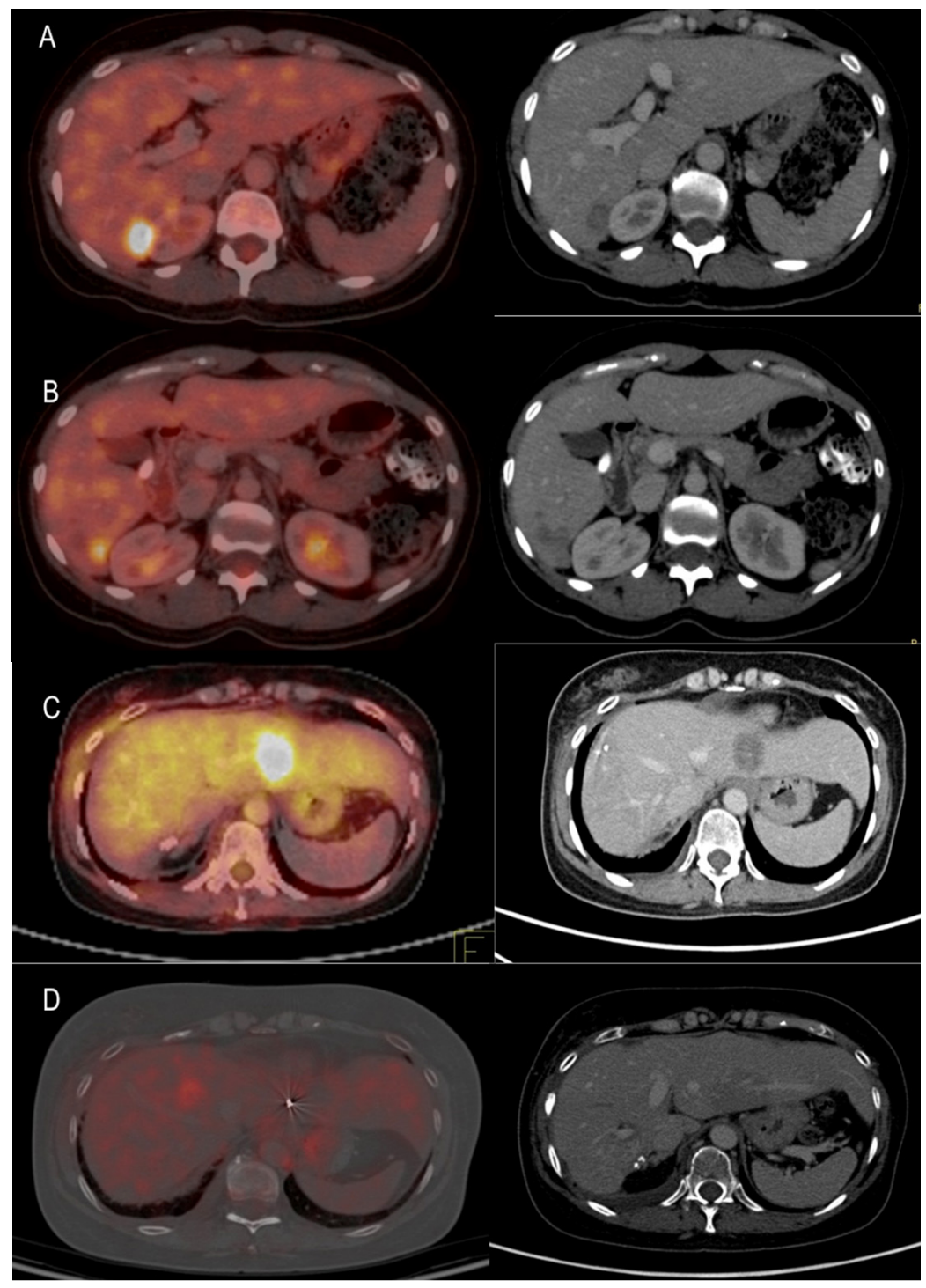

Figure 2. Patient 1: PET-CT scan. A: Sep 2014: $2 \mathrm{~cm} \times 1.6 \mathrm{~cm}$ mass in seg VII of liver; B: Sep 2016: $3 \mathrm{~cm} \times 2.4 \mathrm{~cm}$ mass in seg VIII of liver; C: March 2018: $2 \mathrm{~cm} \times 1.5 \mathrm{~cm}$ mass in seg VI of liver; D: June 2018: PET scan showing complete resolution of mass lesions

VII suggestive of metastasis. There was no other focus of distant metastasis in any other organs. Biopsy from liver lesion was IDC Gr III, ER/PR +ve, Her2neu -ve. She received multiple lines of systemic therapy (Abraxane, Gemcitabine/Carboplatin, capecitabine, HT), but had partial response to treatment. PET-CT (Feb 2016) revealed multiple small lesions in liver with significant metabolic activity in two residual lesions in segment VI $(2 \times 1.5$ SUVmax -5) and VII $(2 \mathrm{~cm} \times 2 \mathrm{~cm}$, SUVmax -8). In March 2016, she was treated with CK ( $45 \mathrm{~Gy} / 3$ fr to both lesions, $86 \%$ isodose). Then she was on immunotherapy with Ipilimumab (PDL1 antagonist). Follow-up PET scan (June 2016) revealed complete metabolic and anatomic resolution of previous liver lesions as well. PET scan in Oct 2016 showed a new solitary lesion in segment II of liver $(2.3 \mathrm{~cm} \times 2.5 \mathrm{~cm}$, SUVmax -8.0) and re-treated with CK (45 Gy/3 fr, 87\% isodose) [Figure 3]. PET scan (March 2017) showed resolution of liver lesions with signs of regeneration/ hypertrophy of the irradiated liver segments. Patient was evaluated with USG abdomen, liver function test for any signs of RILD and no signs of RILD was found on evaluation. Target (GTV) was contoured on contrast CT scan and MRI scan (T1 contrast and T2 flair) images. Usual PTV margin of $3 \mathrm{~mm}$ given. In re-radiation, where the new lesion is away $(>5 \mathrm{~cm}$ ) from the fiducials, $5 \mathrm{~mm}$ PTV margin was given for setup uncertainties. Overlapping of 
Table 2. Case report of breast cancer with liver metastasis at follow up evaluation and re-treated with radiosurgery

\begin{tabular}{|c|c|c|c|}
\hline Date & Event & Treatment & Investigations \\
\hline Nov 2010 & $\begin{array}{l}\text { Right breast carcinoma } \\
\text { ER/PR + ve, Her2neu -ve }\end{array}$ & \multicolumn{2}{|c|}{$\begin{array}{l}\text { Right BCS (pT1N1M0) - Lumpectomy + SNLB and axillary dissection. } \\
4 \times \text { CEF }+4 \times \text { Docetaxel }- \text { RT- } 50 \mathrm{~Gy} / 25 \mathrm{fr}+10 \mathrm{~Gy} \text {, then Tamoxifen }\end{array}$} \\
\hline Feb 2014 & $\begin{array}{l}\text { Metastatic disease } \\
\text { detected }\end{array}$ & \multicolumn{2}{|c|}{$\begin{array}{l}\text { USG Abdomen - Multiple lesions in Liver. } \\
\text { PET CT - Multiple metastatic deposits in both lobes of liver, largest } 7.6 \mathrm{~cm} \times 9 \mathrm{~cm}-\mathrm{Seg} \text { VI, } \\
\text { VII. } \\
\text { No other focus of distant metastases. } \\
\text { Needle biopsy Liver lesion - Metastatic high grade ductal carcinoma (ER/PR +ve, Her2neu } \\
\text { negative); CA 15.3: } 1291 \\
\text { Started on Abraxane } \times 4 \text { Cycle }\end{array}$} \\
\hline June 2014 & Restaging & \multicolumn{2}{|c|}{$\begin{array}{l}\text { Partial response } \\
\text { PETCT - Significant reduction in no of lesions, metabolic activity and size. CA 15.3 = } 60 \text {. } \\
\text { Started on LHRHA + Anastrozole }\end{array}$} \\
\hline Oct 2014 & Chemotherapy & $\begin{array}{l}\text { Abraxane } \times 3 \text { cycles, } \\
\text { then Gem } / \text { Carbo } \times 4 \text { Cycle }\end{array}$ & $\begin{array}{l}\text { Rising CA } 15.3 \text { = } 39 \text { à } 66 \\
\text { PET CT - Disease progression - Increase in size } \\
\text { and metabolic activity of liver lesion Segment VII } \\
(4.5 \mathrm{~cm} \times 4 \mathrm{~cm} \text {; SUVmax } 6.1) \text {. New metabolic } \\
\text { active liver lesion segment VI }(1.4 \mathrm{~cm} \times 1.4 \mathrm{~cm} \text {; } \\
\text { SUVmax 5.4 ) }\end{array}$ \\
\hline March 2015 & Restaging & Complete metabolic response & $\begin{array}{l}\text { PETCT - Complete resolution of metabolic activity } \\
\text { and reduction in size of lesions in segment VI, VII. } \\
\text { On Tamoxifen }\end{array}$ \\
\hline Aug 2015 & Progression & $\begin{array}{l}\text { Inj Goserlin monthly + Exemestane + } \\
\text { Everolimus } \\
\text { Then on Capecitabine }\end{array}$ & $\begin{array}{l}\text { CA } 15.3=25 \\
\text { PETCT - Disease progression - New small liver } \\
\text { lesions in segment V, VI, VII . No other site of } \\
\text { distant metastases. }\end{array}$ \\
\hline Feb 2016 & Restaging & \multicolumn{2}{|c|}{$\begin{array}{l}\text { PETCT - Significant reduction in size of all lesions. } 2 \text { lesions appear metabolically active - } \\
\text { Segment VI }-1.8 \mathrm{~cm} \times 1.5 \mathrm{~cm} \text {, SUVmax } 4.7 ; \text { Seg VI/VII }-2.2 \mathrm{~cm} \times 2.2 \mathrm{~cm} \text {, SUVmax } 7.6\end{array}$} \\
\hline March 2016 & 1st CK Liver & \multicolumn{2}{|c|}{$\begin{array}{l}\text { SBRT - } 45 \text { Gy in } 3 \text { fractions (prescribed to } 86 \% \text { isodose line) delivered to the two FDG avid } \\
\text { liver lesions } \\
\text { Then on Ipilimumab }+ \text { Nivolumab } \times 7 \text { cycles }\end{array}$} \\
\hline July 2016 & Restaging & \multicolumn{2}{|c|}{$\begin{array}{l}\text { CECT scan - resolution of all lesions, except lesion in Seg VI/VII which has substantially } \\
\text { reduced in size. }(2 \mathrm{~cm} \times 1.2 \mathrm{~cm})\end{array}$} \\
\hline Nov 2016 & $\begin{array}{l}\text { 3rd CK Liver } \\
\text { CK - Brain }\end{array}$ & \multicolumn{2}{|c|}{$\begin{array}{l}\text { MRI Brain }-2.3 \mathrm{~cm} \times 2.8 \mathrm{~cm} \times 3.2 \mathrm{~cm} \text { in left premotor region. CK } 27 \mathrm{~Gy} / 3 \mathrm{fr} \\
\text { PET-CT - Disease Progression }- \text { New FDG avid lesion in left lobe of liver }- \text { Segment II }(2.3 \mathrm{~cm} \\
\times 2.5 \mathrm{~cm} \times 2 \mathrm{~cm} \text {, SUVmax } 8.0 \text { ) } \\
\text { SBRT to Segment II liver lesion }-45 \mathrm{~Gy} \text { in } 3 \text { fractions, (prescribed to } 87 \% \text { isodose line). }\end{array}$} \\
\hline March 2017 & Restaging & \multicolumn{2}{|c|}{$\begin{array}{l}\text { PET-CT - Disease Progression - Segment II lesion - CR. Three new FDG avid poorly enhancing } \\
\text { ill defined lesions appeared Seg IVa }(1.1 \mathrm{~cm} \times 1.3 \mathrm{~cm} \text {, SUVmax } 4.2) \text {, Seg III/IV }(1.8 \mathrm{~cm} \times 1.3 \mathrm{~cm} \text {, } \\
\text { SUVmax 8.0), Seg VI }(1.2 \mathrm{~cm} \times 1.2 \mathrm{~cm}, S U V \max 5.8) \\
\text { FDG avid small lesion on left side of sacrum near neural foramina. }\end{array}$} \\
\hline April 2017 & 4th CK & \multicolumn{2}{|c|}{ Dose of 25 Gy in 5 fractions to sacral lesion } \\
\hline
\end{tabular}

FDG: fluorodeoxyglucose; SNLB: sentinel lymph node biopsy; BCS: breast conservative surgery; SUV: ; CECT: ; CEF:

previous dose distribution was evaluated. No "hot spot" (more than prescribed dose) outside the target volume was seen. Follow-up PET scan (Oct 2017) showed extensive metastasis in other organs. She was on metronomic chemotherapy and expired with progressive disease on June 2018.

\section{DISCUSSION}

Re-radiation in liver tumours are not common in clinical practice. There are only few published literatures in this aspect and no standard consensus regarding dosage schedule ${ }^{[6-8]}$. In most of the subsites, such as in head and neck cancer or cervical cancer, in re-irridiation setting there is usually reduction of total dose $(\mathrm{BED})^{[9]}$. Treatment volume is limited and fractionation schedule modified depending upon "time to re-treat". Irridiated volume is also important in selection of fractionation schedule ${ }^{[8]}$. Usually, in head and neck cancer "seven" year time is considered "safe" to re-challenge with full dose of RT. In case of reradiation before that period, there is a reduction of dose depending upon the "time to re-treat". Usually $15 \%$ dose "decay" considered in 1st year after RT and then every year 10\% "decay" in dose. As the time gap between primary RT and re-irridiation is increasing, it's safer to deliver higher (adequate) dose of RT 


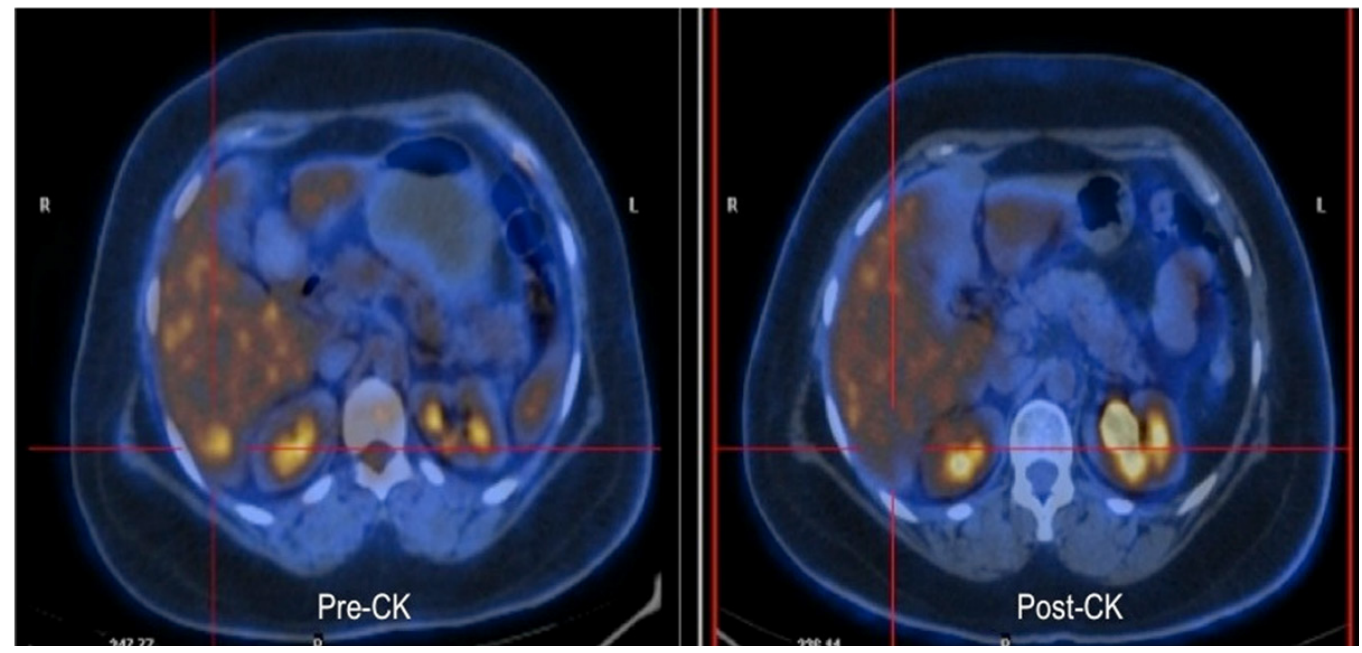

Figure 3. Patient 2: PET scan uptake showing response to treatment. Pre-CK: pre-cyberknife; Post-CK: post-cyberknife

to the target ${ }^{[9]}$. This standard practice is not applied in re-radiation of liver tumours. In fact, in few studies there are better results (OS) in patients treated with higher dose in re-radiation setting. Child Pugh Score and "time to re-treat" are considered significant prognostic factors ${ }^{[6]}$. There is no compromise in irridiated volume as well ${ }^{[6]}$. Tolerance of liver is low, but fortunately in re-radiation setting, liver tolerates radiation comparatively better than other subsites ${ }^{[2,5]}$. High dose RT work like thrombo-embolism, embolizing blood supply to a portion of liver and stimulating proliferating of hepatocytcyes from adjacent normal liver ${ }^{[7]}$. Proliferating hepatocytes cause hypertrophy of the liver portion which is naiive to $\mathrm{RT}^{[7]}$. This proliferating hepatocytes replace the post-CK necrotic liver. Hence, the "new" regenerated portion of liver tolerate better than previously treated liver. Different cytokines liberated from the necrosed liver tissue may also stimulate hypertrophy of liver. It is assumed that the new hepatocytes are naïve to RT and will tolerate radiation better. However, there is no prospective study neither any laboratory model to establish this notion.

After RT, there is fibrosis as well, and this fibosis may lead to shrinkage of liver volume. Post-CK, there is $50 \%$ regression of the involved liver due to radiation injury, on the other hand there is $320 \%$ compensatory hypertrophy of the contralateral liver lobe ${ }^{[2]}$. This phenomenon negates the implications of firbosis, and hypertrophy has more predominent impact. Shrinkage of liver volume is expected to be more with higher integral dose of RT. In few studies, there is transient reduction of liver volume of about $20 \%$ at 3 months post-CK. However, at one year follow up there is only $10 \%$ shrinkage compared to pre-treatment volume. Even after repeating CK, liver volume is mostly maintained due to compensatory hypertrophy.

Most severe complication after re-radiation is $\operatorname{RILD}^{[7,8]}$. It is a syndrome of ascites, elevated transaminase level, and anicteric hepatomegaly. Usually occurs in a proportion of patient after receiving whole liver doses of $>30-35 \mathrm{~Gy}^{[8]}$.

However, retrospective series of partial liver radiation have demonstrated that liver tolerance not only depends upon the total dose of RT, but also on pre-treatment Child-Pugh score, viral load and volume of tumour as well ${ }^{[8]}$. Partial liver may be safely treated with radiation if adequate liver volume is preserved ${ }^{[3]}$. In re-radiation, as the hypertrophied liver is mostly radiation naiive, re-radiation is possible with adequate dose in small volume recurrences.

In this present case series, breast cancer patients with liver metastasis were treated with radiosurgery for multiple times in recurrent setting. There was a time gap of more than six months between two treatments. 
There was no clinical sign of "RILD" after re-CK. Radiological evaluation, clinical examination and liver function test done to exclude RILD at all post-CK follow up evaluation. In both the cases, liver volumes were maintained after post-CK long-term follow up ( $>2$ years). There was regeneration of the treated portion of liver. In the present series, patient was treated three times with $\mathrm{CK}$ for liver metastasis. There are few small series of liver metastasis patients treated with SBRT for twice in two different lobes. There is limited or no published literature on SBRT for three times in liver metastasis. In present study is novel in terms of thrice CK treatment for liver metastasis and had complete response to treatment. Modern systemic therapy improves the probability of control of distant metastasis as well as survival ${ }^{[9]}$. Hence, the probability of repeating focal treatment with RT has increased significantly with usage of modern systemic therapy. Focused RT with Robotic Radiosurgery (CK) has minimal internal target volume and spares maximum liver volume, hence enables to re-treat $\mathrm{w}$ ith radiosurgery in small volume recurrent or new lesions in liver. Toxicity was assessed by liver function test parameters, ascitis and clinical symptoms ${ }^{[8]}$. There was no gross derangement of secretory or excretory functions (serum bilirubin, alkaline phosphatase, SGOT, SGPT) of the liver. There was no ascitis after treatment or at follow up evaluation. Patients were asymptomatic with liver metastasis and were on routine close follow up Usually, after liver metastasis survival outcome is poor, mean overall survival is six to eight months after diagnosis. In this case series, both the patients survived more than 2 years and after CK there is acceptable survival outcome in these patient cohort.

In recent years, with advent of modern more potent systemic therapies as second and third line treatment, possibility of re-radiation of liver metastasis has increased ${ }^{[9,10]}$. RT for liver metastasis at diagnosis and at follow up evaluation is more common and needs to be addressed. Long-term survival ( $>2$ years) is seen in breast cancer patients with liver metastasis and also in known patients with liver metastasis on routine follow up evaluation.

In summary, re-radiation for liver lesions is feasible but uncommon in clinical practice. In the present series, two patients with liver metastasis were treated three times with radiosurgery for metastasis at different segments of liver without any clinical signs of liver decompensation. There were are signs of early regeneration in the irradiated regions of the liver in USG scan. High regeneration capacity and hypertrophy of the irradiated region of liver suggest potential for Re-RT. Re-radiation of liver with CK will be an exciting option in the era of highly potent systemic therapies.

\section{DECLARATIONS}

\section{Authors' contributions}

Concept and design: Dutta D, Krishnamoorthy S

Data analysis and interpretation: Dutta D, Krishnamoorthy S, Nair H

Manuscript preparation: Dutta D, Das R, Madhavan R, Holla R

Critical review and finalization of the manuscript: Dutta D

\section{Availability of data and materials}

Not applicable.

\section{Financial support and sponsorship}

Not applicable.

\section{Conflicts of interest}

All authors declared that there are no conflicts of interest.

\section{Ethical approval and consent to participate}

Not applicable. 


\section{Consent for publication}

Not applicable.

\section{Copyright}

(c) The Author(s) 2019.

\section{References}

1. Seol SW, Yu JI, Park HC, Lim DH, Oh D, et al. Treatment outcome of hepatic re-irradiation in patients with hepatocellular carcinoma. Radiat Oncol J 2015;33:276-83.

2. Farach A, Quesada J, Teh BS. Liver regeneration following repeat SBRT. J Gastrointest Oncol 2015;6:E2-E6.

3. Dawson LA. Ten Haken RK. Partial volume tolerance of the liver to radiation. Semin Radiat Oncol 2005;15:279-83.

4. Jackson A, Marks LB, Bentzen SM, Eisbruch A, Yorke ED, et al. The lessons of QUANTEC: recommendations for reporting and gathering data on dose-volume dependencies of treatment outcome. Int J Radiat Oncol Biol Phys 2010;76:S155-60.

5. Reed GB Jr, Cox AJ Jr. The human liver after radiation injury. A form of veno-occlusive disease. Am J Pathol 1966;48:597-611.

6. Høyer M. Re-irradiation with stereotactic body radiation therapy (SBRT). Chin Clin Oncol 2017;6:S15.

7. McDuff S, Remillard K, Wo JY, Raldow A. Safety and Tolerability of Liver Re-Irradiation. J Radiat Oncol Biol Phys 2017;99:E171.

8. Raldow A, Wo JY, Wolfgang JA, Zhu AX, Hong TS. Tolerability of Liver Re-irradiation With Stereotactic Radiation Therapy. J Radiat Oncol Biol Phys 2014;90:S374.

9. Caudell JJ, Ward MC, Riaz N. Volume, dose and fractionation consideration for IMRT-based reirradiation in head and neck cancer: A multi-institutional analysis. Int J Radiat Oncol Biol Phys 2018;100:606-17.

10. Yoo TG, Cranshaw I, Broom R. Systematic review of early and long-term outcome of liver resection for metastatic breast cancer: Is there a survival benefit? Breast 2017;32:162-72. 